\title{
Adaptive scans allow targeted cell-ablations on curved cell sheets.
}

\author{
Huicheng Meng ${ }^{1}$, Dmitry Nuzhdin ${ }^{1}$, Miguel Sison ${ }^{1}$, Frédéric Galland ${ }^{1}$, and Loïc LeGoff ${ }^{2, *}$ \\ ${ }^{1}$ Aix Marseille Univ, CNRS, Centrale Marseille, Institut Fresnel, Marseille, France. \\ ${ }^{2}$ Aix Marseille Univ, CNRS, Centrale Marseille, Institut Fresnel, Turing Center for Living Systems,Marseille, France \\ *Corresponding author: Loïc LeGoff (loic.le-goff@univ-amu.fr)
}

\section{ABSTRACT}

Tissue morphogenesis proceeds through the mechanical actuation of cells by active forces from the cytoskeleton. Multicellular laser ablation has emerged as an essential means to probe these active forces by severing the tissue locally and inferring the tension born by the severed domain from the recoil of the surrounding structures. The realm of applications of laser ablations is however limited by the 2D steering of the laser in most instrumental configurations, while embryos and developing tissues are intrinsically 3D structures. Here, we present a flexible near infrared (NIR) fs-pulsed laser ablation system in which ablation trajectories proceed in 3D and adapt to the curved surface of cell sheets, which are prominent structures in embryos. Trajectories are computed through an unsupervised search for the surface of interest. We demonstrate that, depending on the exact experimental setup, the surface estimation can rely on a high content 3D imaging with a combined confocal microscope, or alternatively on a rapid Lissajou scan of the sample space with a NIR stand-alone setup. We apply the developed strategy mapping tensions in the developing Drosophila wing imaginal disc. These targeted, adaptive scans could be applied to other forms of non-linear processes such as two-photon fluorescence imaging or opto-genetics.

\section{Introduction}

Our understanding of morphogenetic processes has considerably improved in the past decade owing to a mutlidisciplinary effort to address biological shapes in mechanical terms. A key element is to relate tissue-distortions and flows with the pattern of force generation imposed by active cytoskeletal elements. This new approach to investigate tissues was made possible by several technical implementations. First, improvements in live imaging allow us to image entire embryos at a subcellular resolution $^{1}$, and to quantify accurately strains and flows within the tissues. Genetically encoded fluorescent proteins give us a glance at the cytoskeletal activity giving rise to force generation ${ }^{2}$. Second, one can now probe mechanical stresses within tissues. This can be done in varying experimental contexts using traction force microscopy ${ }^{3,4}$, Force-inference methods ${ }^{5-7}$, FRET sensors ${ }^{8,9}$, micro-droplets and microbeads stress sensors ${ }^{10,11}$ or laser ablations ${ }^{12-14}$. Third, one can locally perturb patterns or force generation with light using optogenetics ${ }^{15,16}$ and force transmission using laser ablations ${ }^{17-19}$.

Among the aforementioned techniques, laser ablation (LA) has emerged as a widespread method to quantify mechanical properties as it is easy to implement in vivo and in situ. The working principle of LA is to locally cut the biological tissues using either UV light or fs-pulsed near infrared light (NIR). Upon local disruption, the amplitude and speed of the recoil instructs on the mechanical stress that was born by the ablated region ${ }^{20}$. In essence, laser ablations recapitulate at a microscopic scale and without the need for direct physical access the established technique of hole-drilling residual stress measurements ${ }^{21}$, a technique used, for example, more than 150 years ago by Langer to investigate tension of the human skin $^{22}$.

Tissues can be ablated in a pointlike fashion to disrupt organelles and the cytoskeleton or along small lines or circles. The size of the ablation depends essentially on the size of the structure to be disrupted or on the characteristic size at which tissue mechanics must be probed. Changes in the shape of tissues are usually well described by continuum modeling approaches at spatial scales larger than the cell. In this context, it is important to probe tissue mechanics at mesoscopic scale, which is why multi-cellular ablations have been developed using circle or line ablations ${ }^{23,24}$.

A major difficulty in multi-cellular ablations lies in the fact that embryos and developing tissues are usually curved. Laser scanning systems, which steer the laser focus in 2D, cannot comply with the need for a precise 3D trajectory along the surface of the tissue to ensure an even degree of cutting along the ablation trajectory. This is all the more problematic in multi-photon systems where the non-linearity of plasma induction makes it highly sensitive to the distance between the ablated structure and the laser focus. Embryonic tissues are often organized in cell sheets, called epithelia, which take a central role in structuring embryos and precursors of adult tissues ${ }^{25}$. A lot of the forces underlying morphogenesis concentrate at the apical and basal surfaces of these cell sheets, which are site of enrichment in the force-generating cytoskeleton. Whether ablating the apical or 
the basal side, the trajectory of the laser focus must precisely follow the 3D contour of the surface of interest in order to avoid artefacts in the mechanical measurements linked to uneven ablation. The time needed to complete the trajectory should also be shorter than the relaxation time of the tissue, which can be complicated in a fully 3D context, as steering-speed is constrained by peak power, pulse-energy and repetition rate of the laser. One solution used to avoid these problems is to flatten the tissue mechanically. This, however, inevitably alters stress distribution and should be avoided as much as possible. What is needed is an ablation system which can perform 3D ablation trajectories that adapt accurately to the surface of the cell sheet. Such an adaptive laser-scan would maintain targeted structure always at the center of the laser focus.

In this paper, we present a flexible NIR fs-laser ablation system to investigate cell and tissue mechanics. In this system, ablation trajectories proceed in 3D and adapt dynamically to the curved surface of epithelial cell sheets (Fig. 1). Trajectories are computed through an unsupervised search for the surface of interest. This system allows to perform multicellular ablations on curved epithelia, such as the Drosophila wing imaginal disc, where conventional 2D systems fail to proceed.

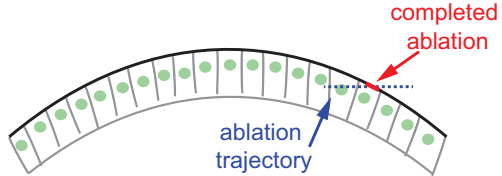

a 2D ablation

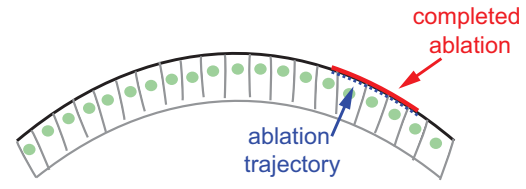

b Adaptive ablation

Figure 1. Ablating a curved cell-sheet. (a) In a conventional ablation system the laser focus is scanned along a $2 \mathrm{D}$ trajectory which will miss regions of the structure to ablate that are above and below the focal plane of the microscope objective. (b) In an adaptive ablation system, synchronization of the $\mathrm{z}, \mathrm{x}, \mathrm{y}$ steering of the laser ensures that a 3D ablation trajectory accurately follows the contours of the surface of interest.

\section{Results}

\section{Experimental set-up}

The custom built setup comprises two optical paths, one for imaging and one for ablation (see methods and Fig. 2a). Briefly, imaging is performed with a spinning disc confocal microscope using $488 \mathrm{~nm}$ and $561 \mathrm{~nm}$ lines. A fs-pulsed NIR laser (930 nm) is focused in the imaging plane by the objective and steered by galvanometer mirrors placed in a plane conjugated to the back focal plane of the objective. A piezo-actuated stage ensures z-translation of the sample synchronously with the $\mathrm{x}, \mathrm{y}$-steering from the scanner to provide 3D trajectories. A data acquisition board outputs properly timed analog signals for x,y,z-translations and their synchronization with the camera and on/off modulation of the lasers. All hardwares are controlled using Matlab for maximum flexibility. Specifically, imaging hardwares are controlled within matlab through the $\mu$ Manager core $^{26}$. 
a

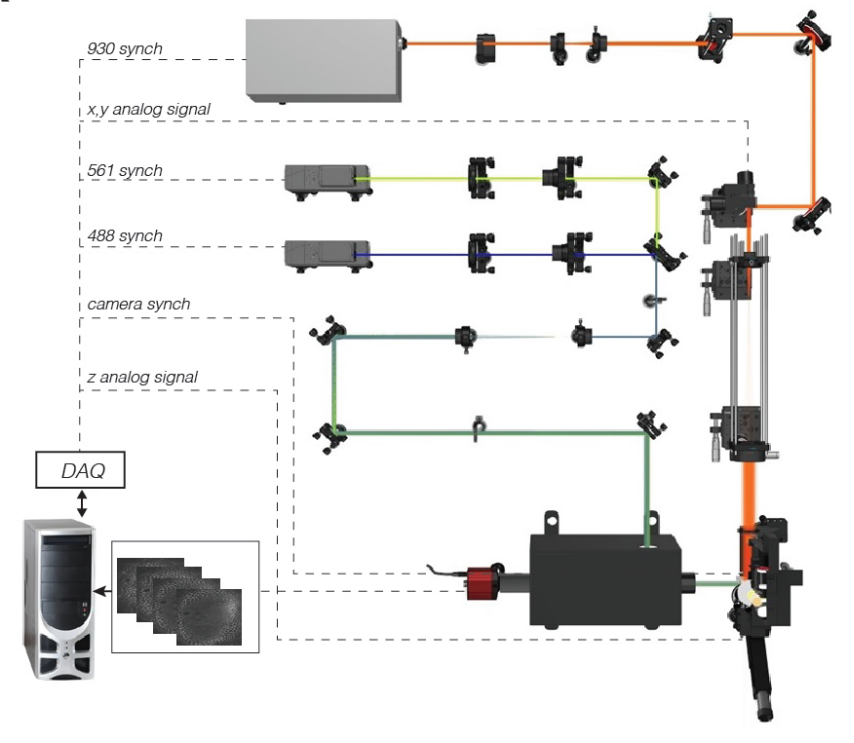

d

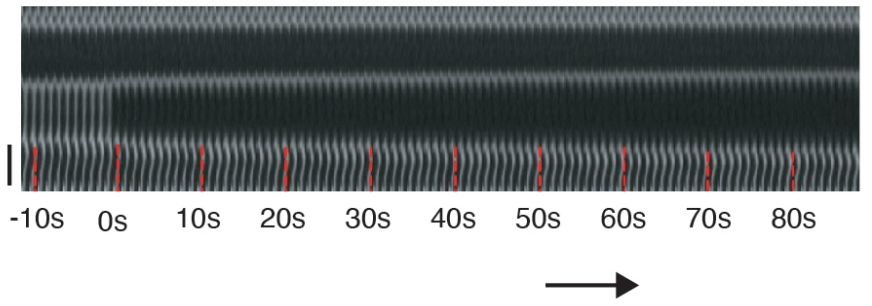

time after ablation b
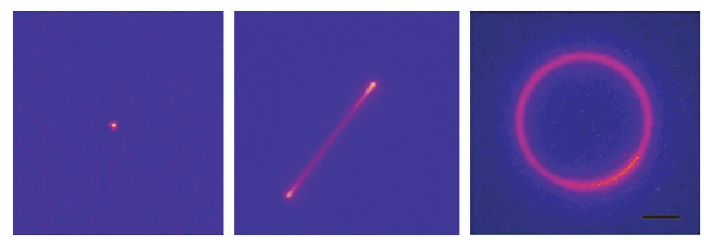

C

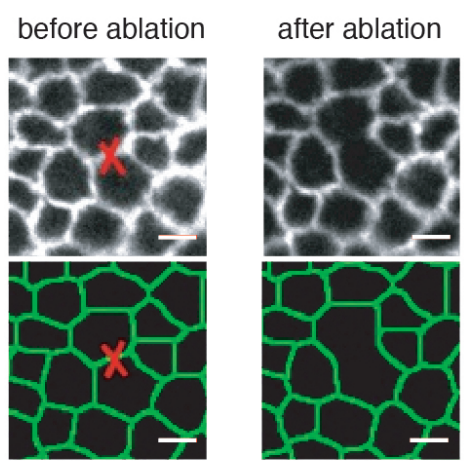

e

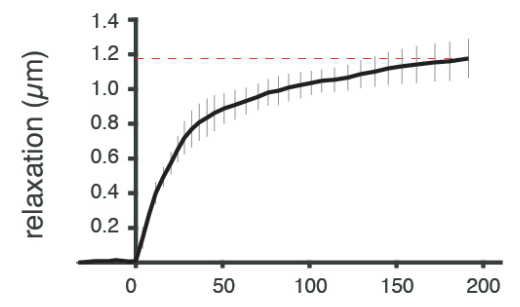

time after ablation (s)

Figure 2. Optical setup for the combined imaging and ablation of epithelial tissues. (a) Optical layout. (b) Modes of fs-pulsed laser steering for ablation; from left to right : points, line, circles. (c-e) Example of an ablation a the single junction scale. (c) Before/after comparison of the tissue around the severed junction (indicated by a red cross). Cell junctions are visualized through the imaging of a cadherin:GFP fusion. The bottom panel shows the segmented cell outlines. (d)Relaxation dynamics of the severed junction as observed through a kymograph. (e) Quantification of the relaxation. All scale bars are $3 \mu \mathrm{m}$

The resulting apparatus performs 2D scanning ablations in a similar fashion to previously published works ${ }^{12,14,17,23,24,27}$. Ablations can be performed in a point like fashion, or along lines and circles (Fig. 2b). A typical experiment is illustrated on Fig. 2c-e. Individual cell-cell epithelial junctions are severed through a point or a small line ablation (Fig. 2c). As a result of the ablation, the junction cannot bear tension anymore. A mechanical relaxation of the surrounding tissue follows (kymograph in Fig. 2d). The relaxation is quantified by measuring the distance between the two tri-cellular junctions connected by the severed junction (Fig. 2e). Notably, the initial slope is often used as a proxy for $T / \zeta$, where $T$ is the tension initially born by the junction and $\zeta$ is the effective friction of the medium ${ }^{28}$.

\section{D vs 3D ablations}

Developing tissues are often curved structures. Such is the case, for example, of the Drosophila wing imaginal disc used in this study $^{29,30}$-an important model system to study the regulation of growth and morphogenesis ${ }^{31}$. In this context, a difficulty for the mechanical characterization of tissues arises when performing ablations at intermediate to large scales, as the curvature of the tissue hinders the ablation process over large distances when the laser is steered in 2D. We could perform multi-cellular ablations at the apex of the tissue - provided the tissue is sufficiently flat and horizontal there (Fig. 3a-d). The ablation trajectory then follows the contours of the tissue (red circle in Fig. 3a). When using the pulsed laser at low power to perform two photon imaging ( $<50 \mathrm{~mW}$ after the objective) the fluorescence of the junctional marker (a cadherin:GFP protein fusion) is excited homogeneously along the circular trajectory (Fig. 3b). A complete circular ablation proceeds if we then scan the laser at high 
power ( $\sim 165 \mathrm{~mW}$ after the objective) along the same trajectory (Fig. 3c,d). Fully successful circular ablations, however, are limited to a small apical region of the tissue. In the curved regions, the 2D ablation trajectory cannot follow the contours of the tissue (red circle in Fig. 3e). We observe that the emitted fluorescence of the junctional marker is non-homogeneously distributed when we scan the trajectory at low laser-power (arrows in Fig. 3f). Ablation relies on a five-photon ionization process $^{32}$, which makes it even more sensitive to a proper localization of the laser focus with respect to the adherens plane. We indeed observe only partial ablations (arrows in Fig. 3g,h) when scanning a high power laser along the 2D circular trajectory. Ablation is effective in only a fraction of the trajectory (arrows in Fig. 3h).
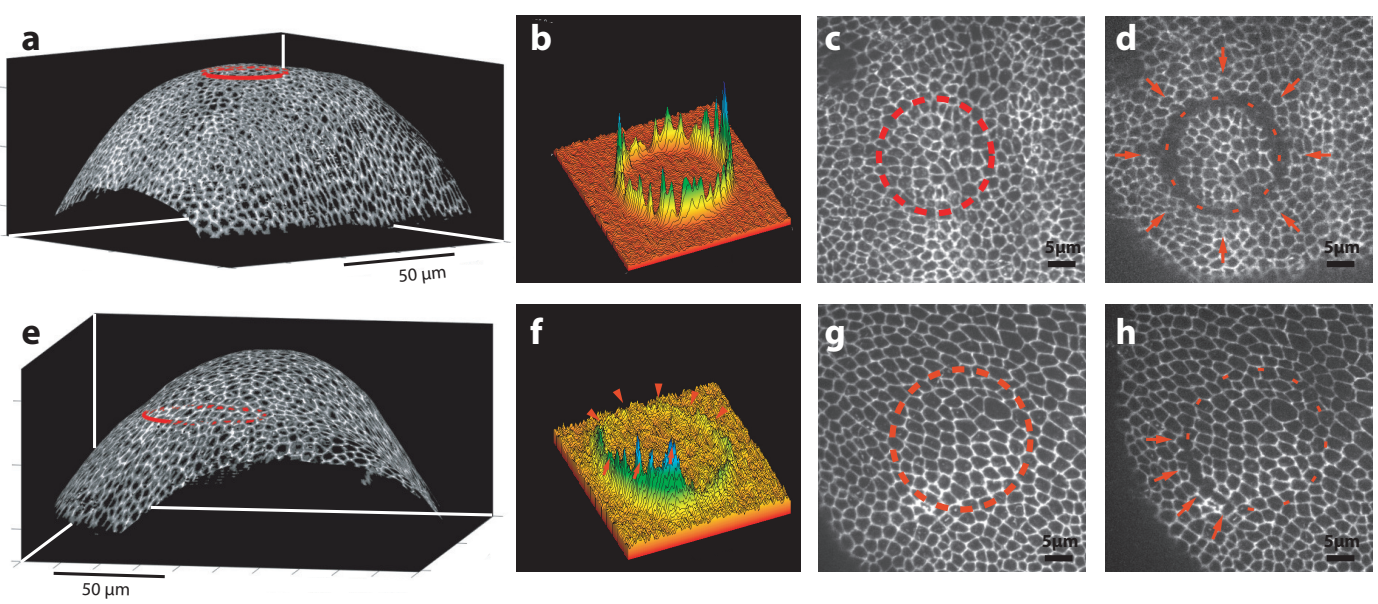

Figure 3. Failed circular ablation on curved tissues. (a-d): 2D ablations along the flat apex of the tissue are successful. (a): 3D topography of a curved wing imaginal disc (cadherin:GFP labeling). A 2D circular trajectory (red line) can follow the contours of the tissue. (b): At low excitation power, the laser can excite GFP-fluorescence of the junctional marker on the whole trajectory through a two-photon excitation effect. $(\mathrm{c}, \mathrm{d})$ : Using the same laser along the same trajectory, but at high power, generates an ablation on the whole trajectory (red arrows). (e-h): 2D ablations along the slopped regions of the tissue fail. (e): The 2D circular trajectory does not follow the contours of the adherens surface. (f): At low excitation power, the laser excites fluorescence of the junctional marker only partially, failing to do so in large portions of the trajectory (red arrowheads). $(\mathrm{g}, \mathrm{h})$ : The laser at high power along the same trajectory ablates the tissue in a fraction of the trajectory (red arrows), failing to do so elsewhere.

We implemented a 3D ablation system to overcome the observed failure of multicellular ablations with a planar trajectory (Fig. 4). The principle is first to estimate the surface of the epithelium $Z_{s}(x, y)$ through image analysis (see details below and in the methods). We then generate a trajectory $(x(t), y(t), z(t))$, enforcing that it lies on the computed surface $Z_{s}(x, y)$. In the course of ablation, the z-component of the trajectory is generated by moving the sample stage in synchrony with the xy-actuation from the galvanometric mirrors (Fig. 4a,b). The generated trajectory (Fig. 4c), accurately follows the contours of the epithelium surface (Fig. 4d). With such a process, we could reproducibly perform multi-cellular ablations in slopped regions of the wing imaginal disc (Fig. 5a,b). When scanning the NIR laser at low power in a circular trajectory on the surface, the emitted fluorescence (via a two-photon effect) is homogeneous along the trajectory (Fig. 5c). This reveals that the focus of the laser remains in the plane of the labeled junctional components (E-cadherin:GFP) throughout the 3D-scan. At high laser power, the induced cut is also performed in a homogeneous fashion along the trajectory (Fig. 5b,d). When performing circular ablations, the trajectories are made circular locally along the tangent plane of the surface. To do so, we delineate the circle of given radius on the local tangent, and project this circle on the surface along the normal direction to ensure maximal confocality of the ablation trajectory and the surface (see methods and Fig. S3). 

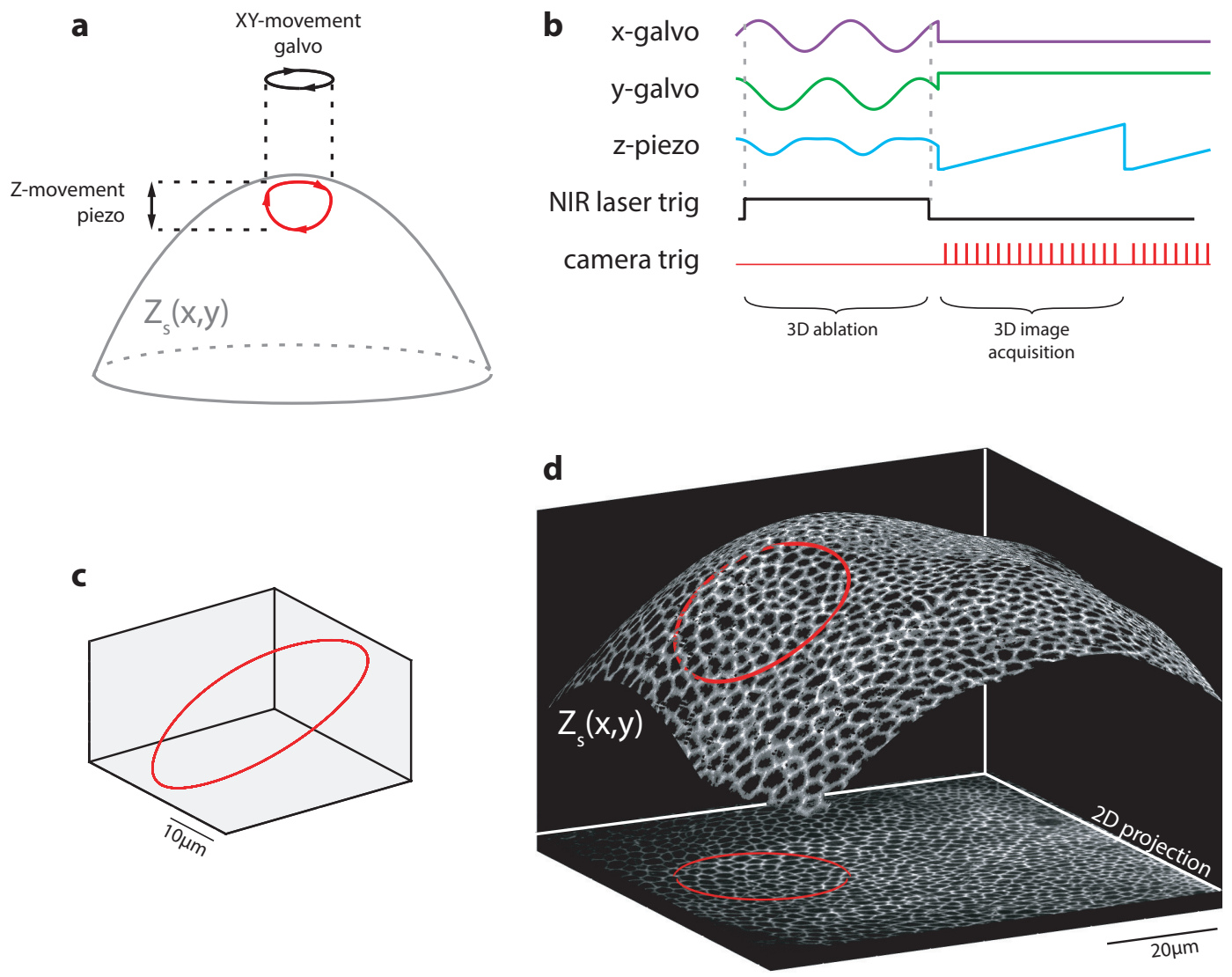

Figure 4. Principle of 3D ablations. (a) A trajectory $(x(t), y(t), z(t))$ is computed so that it lies on the estimated surface $Z_{s}(x, y)$. XY-translation is performed with galvanometric scanners, while Z-translation is performed with the translation stage of the microscope, or alternatively a focus control such as a deformable mirror or an electrically tunable lens. (b) Experimentally, the 3D trajectory relies on the synchronous actuation of $x, y, z$ translation as well as shutter of the NIR laser. The ablation phase is followed by a fast 3D imaging phase to measure the displacements induced by the ablation. (c): example of a 3D trajectory $(x(t), y(t), z(t))$. (d): The 3D trajectory follows the contour of the epithelium surface.

\section{Rapid surface estimation with non-linear Lissajous scans}

The proposed 3D ablations rely on an estimation of the epithelial surface $Z_{s}(x, y)$. So far, the surface has been estimated from the data-rich stacks of a spinning disc confocal (Figs. 3,5). To make our method as general as possible, we designed a strategy for surface estimation which can also be used in the context of stand-alone two photon imaging microscopes -not associated with spinning disc confocal. The single point scanning and lower photon fluxes in non-linear microscopes make imaging in 3D slower, and reduces signal to noise ratio. We solve this problem by performing a very rapid pre-scan of the sample-volume with Lissajous scans. The data is sparse and noisy. This is why we developed a surface estimation method which uses two-photon fluorescence that can work with very few and noisy points derived from the approach proposed in Abouakil et al. for one photon fluorescence ${ }^{30}$.

We briefly present the estimation procedure (see details in the methods section). In a first step, we detect bright points in the volume assuming a normalized intensity distribution for the background after correction of signal inhomogeneities. The estimation then relies on piecewise fits with second order polynomials of the surface that is most densely populated in bright points. These fits are based on RANSAC approach ${ }^{33}$ to be robust to noise on bright points location and fluorophores located outside of the surface. When we estimate only a small portion of the surface (typically the size of a multi-cellular ablation ring $\sim 15 \mu \mathrm{m}$ ), a single polynomial is enough to locally fit the surface (Fig. 6a). When we estimate a large surface (typically the entire epithelial surface), the RANSAC polynomial fits are performed on overlapping windows, and the different reconstructions are fused (Fig. 6b, see methods).

Our method allows to estimate the surface from a small collection of data points. We acquire the necessary 3D data with a relatively slow NIR scanning system, by scanning the sample-space only fractionally. The NIR laser is scanned at low power to 

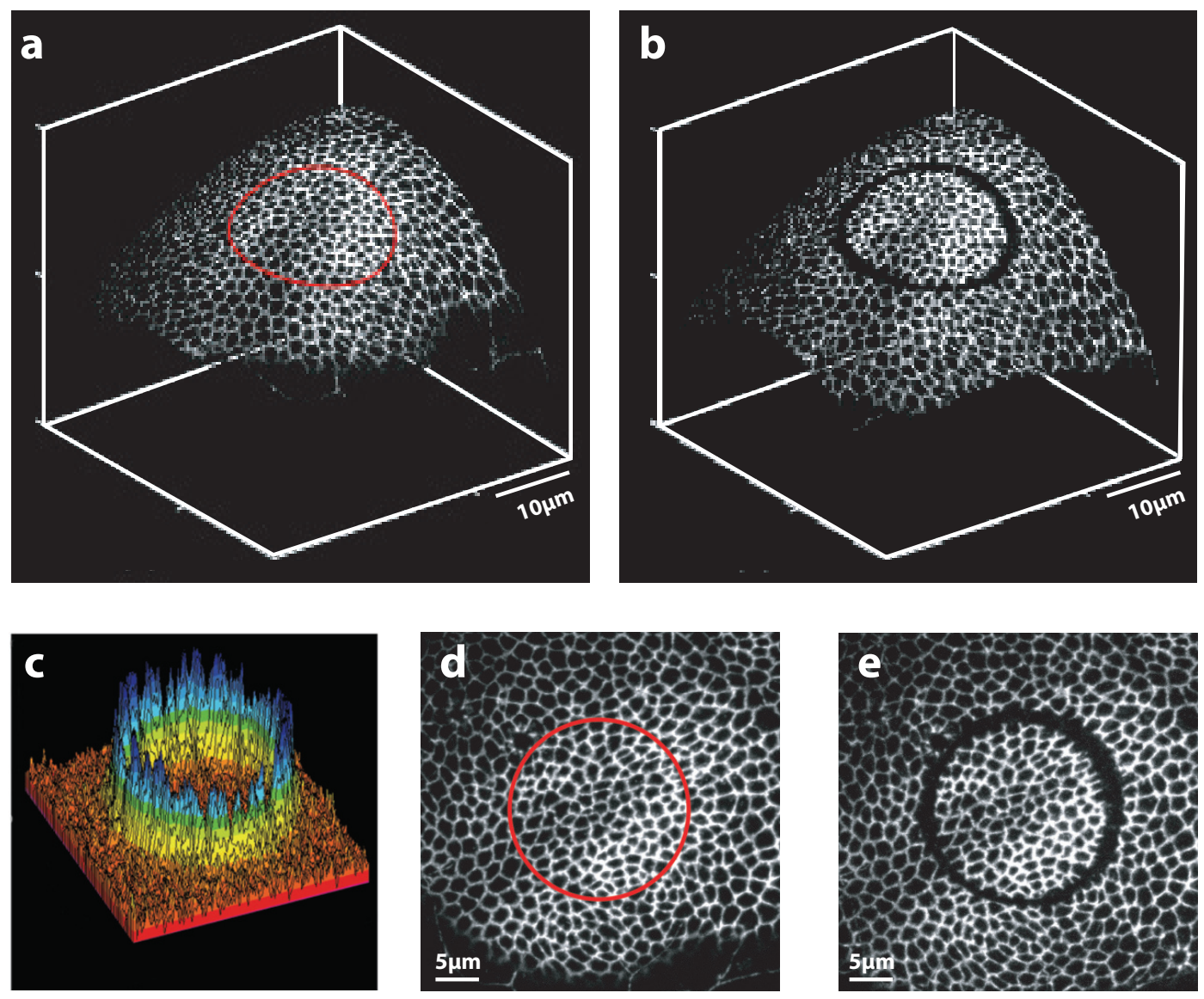

Figure 5. Example of 3D ablations. (a,b) 3D representation of a wing imaginal disc (close up view) before(a) and after (b) a multicellular ablation in a slopped region. (c) At low excitation power, two-photon fluorescence is emitted homogeneously along the $3 \mathrm{D}$ trajectory. $(\mathrm{d}, \mathrm{e}) 2 \mathrm{D}$ visualization of the ablation process showing that ablation has proceeded all along the trajectory.

excite two-photon fluorescence along Lissajous curves (Fig. 6c). Lissajou scans are single harmonic trajectories that do not generate high harmonic components like a raster scan. They are ideal to make fast and fractional scans of the focal plane. Only $8 \%$ of voxels are scanned in the Lissajou trajectories, leading to a more than order of magnitude speed-up with respect to a full, raster scan. Fig. 6d shows the signal obtained form such a Lissajous scan. The structure of interest is hardly recognizable with the human eye. Figs S1a-c shows respectively: i-the detected bright points after normalization of intensities in the sample volume; ii-the most consensual classifications of inliers and outliers from the RANSAC polynomial fits; iii-the surface obtained after interpolation of the inliers. We observed that the estimated surface matches very well with the epithelium. For example, after performing a ground truth full-scan of sample space, and keeping only the signal from a thin shell of thickness $3 \mu \mathrm{m}$ around the estimated surface, a normal cad:GFP image is obtained (Fig. 6e). Thus, the shell could be properly estimated from fast Lissajous scans, despite the fact that non-linear imaging yields a signal with a lower signal to noise ratio than linear spinning disc fluorescence (see Figs. 3,4,5 for comparison). 
a single window fit

- $\bullet$

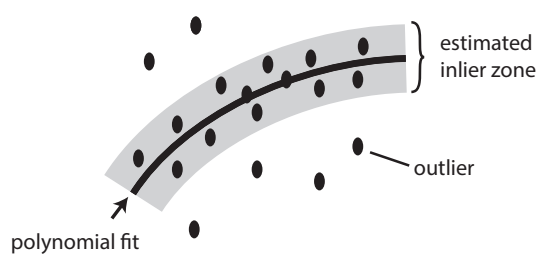

b

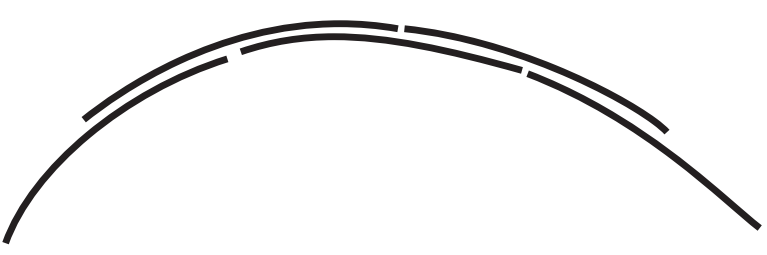

\section{Surface estimation based on rapid Lissajou NIR scans}

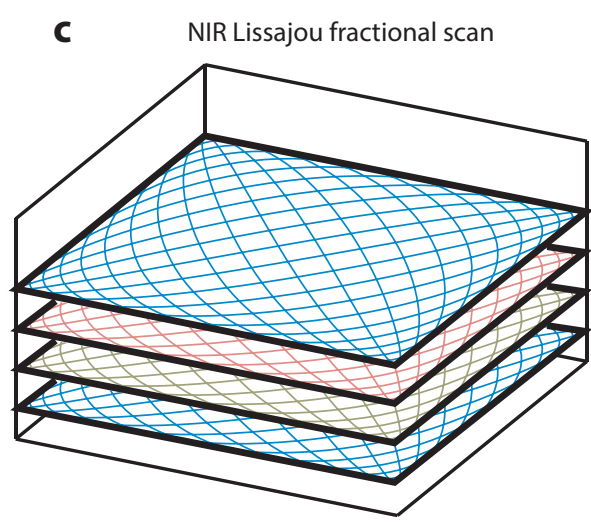

d

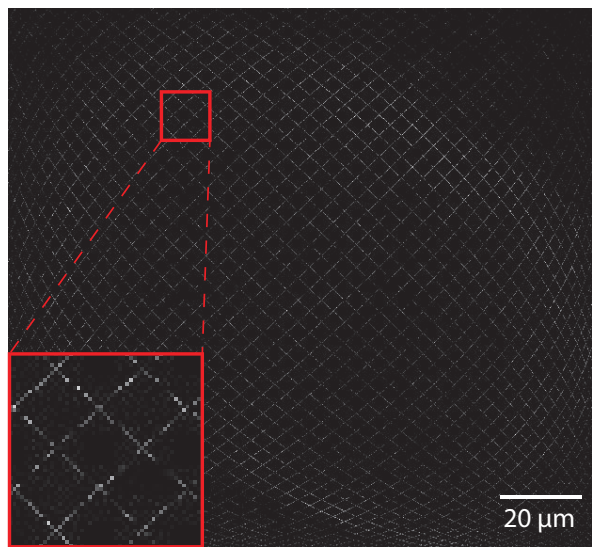

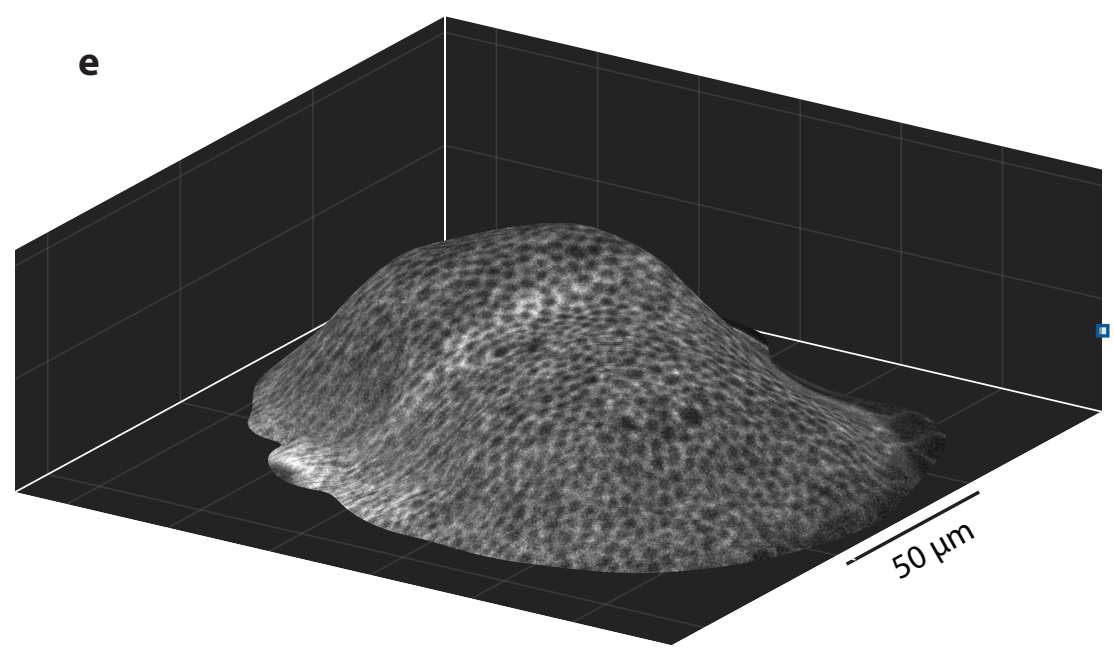

Figure 6. Surface estimation. (a) On short length scales, the surface is fitted with a second order polynomial. With the RANSAC approach, the most consensual fit is obtained by maximizing the number of inliers within a cutoff distance from the fit. (b) On larger length scales, the surface is fitted with multiple orverlapping windows, each window using the RANSAC approach of (a). (c,d) To rapidly estimate the surface of interest using only a NIR laser, we perform a Lissajous scan, which explores a limited number of voxels within the sample space (c: schematic; d: collected fluorescence on one plane). (e) After performing a ground truth full-scan of sample space, we only keep the signal from a thin shell of thickness $3 \mu \mathrm{m}$ around the estimated surface. The fluorescent cad:GFP signal is well encapsulated by the shell.

\section{Application: mapping mechanical asymmetries in the wing imaginal disc.}

We use the ablation setup to explore the distribution of tensions in the wing imaginal discs. The tissue has been shown to be under the influence of a patterned mechanical stress, which influences growth and the organization of the cytoskeleton within 
cells $^{34,35}$. Tensions in the wing imaginal disc have mostly been investigated via single junction, point ablations. Multi-cellular ring ablations offer an attractive alternative as they assess a mesoscopic scale which translates naturally to continuum mechanics and provide robust measurements by averaging the response of multiple cells ${ }^{23,24}$. Most importantly, circular ablations provide a symmetric perturbation which allows to reveal in the clearest manner the asymmetries inherent to the probed tissue: the main axis of relaxation after ablation provides a measurement of the main axis of tension of the tissue, locally. Figure 7a-c show the tissue before and after the ring ablation is performed. The group of cells isolated by the ablation contracts under the influence of its constitutive tension (inner wound margin in yellow on Fig. 7b,c). The outer wound margin (blue on Fig. $7 \mathrm{~b}, \mathrm{c})$ expands orthogonally due to the constitutive tension of the surrounding tissue. The inner and outer margins have their major axis orthogonal from each other. Figure $7 \mathrm{~d}$ maps the displacements of the surrounding tissue following ablation, while Fig. 7e,f quantifies the opening of the wound along the major and minor axis of the outer wound. A detailed analysis of the relaxations after similar annular severings was performed in Bonnet $e t a l .^{23}$. We resort to a simpler analysis focusing only on the asymmetries in tensions: we use the ratio of the slope at origin of the relaxations as a proxy for the ratio the tensions.

We performed ablations in different regions of the wing imaginal disc. Following the results of LeGoff et al., we distinguish peripheral regions (region A in Fig. 7g), medial regions (region B in Fig. . 7g), and the hinge (region C in Fig. $7 \mathrm{~g})^{34}$. The peripheral and hinge regions have been shown to experience an anisotropic stress, while the medial regions are isotropic. We observed characteristic behavior in different regions of the wing disc (Fig. 7h-j). Figure $7 \mathrm{~h}$ shows the response of the tissue in the perriphery. As described in ${ }^{34,35}$, cells are anisotrropic in this region (see the major axis of cells represented by green lines) and globally oriented along the tangent of the tissue. We observe an anisotropic relaxation of the wound, with the main axis of stress qualitatively oriented along the same direction as the cells. To characterize the anisotropy, we measure the ratio of the slope at origin along thet major and minor axis $T_{L A} / T_{S A} \sim 2.4$. In the medial region, the cells are much less anisotropic and a global orientation is hardly visible. The tissue there relax in a fairly symmetric fashion $T_{L A} / T_{S A} \sim 1.3$ (Fig. 7i). In the margin domain (region $\mathrm{C}$ ), the relaxation is also anisotrorpic, with the major axis oriented along the margin, albeit to a less extent than in the periphery $T_{L A} / T_{S A} \sim 1.7$. The anisotropic relaxations at the periphery and at the compartment boundary betray a an anisotorpic stress which is in agreement with previous work ${ }^{34-36}$. Lastly, we stress the importance of the 3D trajectory for the ring ablations. As can be seen on the 3D rendering of Fig. 7k the slopped topography of the tissue required our 3D adaptive scan (see also supplementary movie 1 ). 

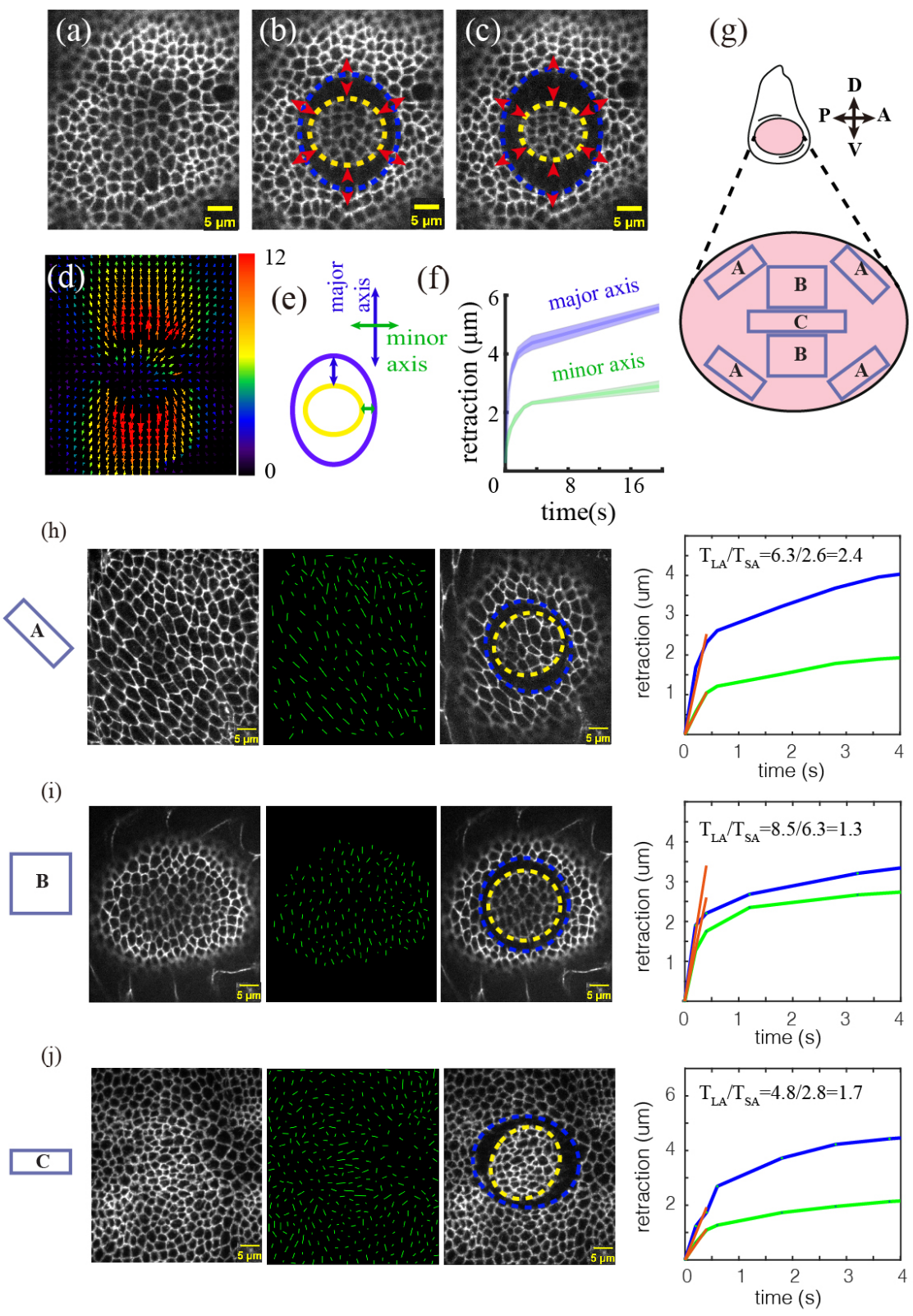

(k)
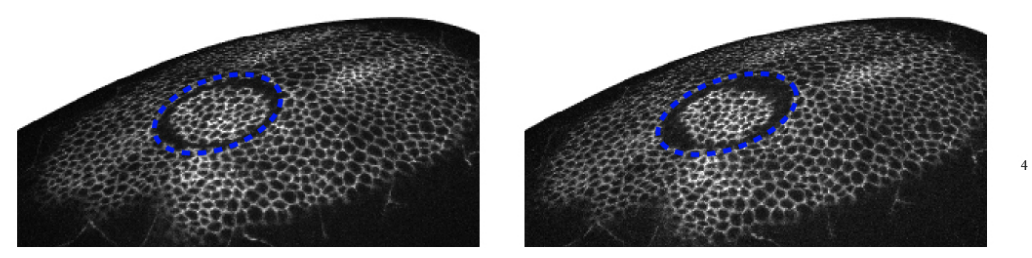

Figure 7. Mapping mechanical asymetries in the wing imaginal disc. (a-f) example of an ablation showing the tissue before (a) and after $(b, c)$ a circular ablation. (d) Displacement map after ablation using particle image velocimetry. (e,f) quantification of the opening of the wound along the outer wound boundary long and short axis: schematic in (e) and measurements in (f). (g) Regionalization of the wing imaginal disc into peripheral (region A), medial (region B) and margin domains (region $\mathrm{C}$ ). The schematic on the left describes where the analyzed presumptive wing tissue (pink) lies with respect to the whole wing imaginal discs. (h-j)Examples of ablations in the mentioned domains. (h) In the periphery, cells are globally anisotropic and oriented along the tangent of the tissue (see green segments indicating the major axis of cells). The main axis of the outer wound is oriented along that same direction, and the response is markedly anisotorpic with a ratio $T_{L A} / T_{S A} \sim 2.4$. (i) In the medial regions, cells are more isotrorpic and a mean orientation does not appear clearly. The relaxations are also more isotropic with a ratio $T_{L A} / T_{S A} \sim 1.3$. (j) In the hinge, despite thet fact that cells are not particularly anisotorpic, the response after ablations is anisotropic $\left(T_{L A} / T_{S A} \sim 1.7\right)$ with the main axis oriented along the margin. (k) 3D representation of the same tissue as (j), at two time points following the ablation. 


\section{Discussion}

We have designed a system to perform precise laser cuts on curved biological surfaces without introducing additional mechanical perturbations. The surface of interest is first estimated using high content 3D-information from a spinning disc confocal. Then the ablation laser is scanned along a 3D trajectory on the surface. We also demonstrate that in the absence of a fast confocal, the surface of interest can be estimated from a rapid Lissajou scan of the NIR laser and two-photon fluorescence collection.

Laser ablations have become a central tool in mechano-biology to infer tensions in tissues from the recoil velocity of the severed structures ${ }^{37}$. In particular, multi-cellular cuts probe mesoscopic scales which are well suited for the confrontation with continuum descriptions, the natural language of mechanics. Circular ablations are particularly convenient as their symmetry allows to reveal directly the anisotropy of tension from the shape of the relaxation. Among the various strategies to generate the cuts, a method of choice is to use NIR, fs-pulsed lasers to make plasma which are well controlled in space. To mitigate the difficulty to perform multicellular cuts on curved tissues, we have introduced an adaptive strategy where the surface of interest is first estimated around the region of interest, and the laser is then scanned along a computed 3D trajectory on the surface. Multi-cellular ablations have already been performed on the wing imaginal disc or other curved tissues ${ }^{36}$. However, it requires a lot of trials and error to position the region of interest within the tissue flat with respect to the focal plane of the imaging objective. We hope our approach will help the generalization of this powerful approach to probe stresses within biological tissues.

While we recently introduced the concept of smart-scans on surface for linear fluorescence ${ }^{30}$, we demonstrate here the concept on non-linear processes for the first time. On an instrumental point of view, the targeted illumination of Abouakil et al. relied on a spatial light modulator in a wide-field optical configuration. Here, the 3D illumination path relies on the control of the scan path of a single point galvo-based scanning microscope. In the current configuration, our set-up performs 3D scans by synchronizing a rather slow z-stage with the fast xy-galvanometric scans. Faster z-scans could be achieved through the use of of an electrically tunable lens ${ }^{38}$ or a deformable mirror ${ }^{39}$.

Many NIR fs-pulsed laser are only available in stand-alone non-linear imaging setups. There, the single point scans and the low photon flux of non-linear contrasts make the 3D-scan of the complete tissue problematically slow, as the tissue may move by the time the volume is scanned and the surface estimated. We thus adapted the experimental approach using Lissajous scans of the NIR laser to rapidly probe the global features of the tissue and estimate the surface from this fractional scan. Lissajous trajectories are optimal to scan space fractionally at high speed as they don't introduce unnecessary high harmonics like raster scans $\mathrm{do}^{40,41}$. Beyond ablation experiments, the proposed adaptive scans could be used in a non-linear imaging context, where restriction of the scan path to a thin shell around the surface of interest could be an efficient means to reduce the light dose and speed up imaging, providing a generalization of the geometry-driven scans of Olivier et al. ${ }^{42}$.

\section{Material and methods}

\section{Spinning disc imaging}

The laser ablation plateform combines a spinning disc fluorescence microscope with a fs-pulsed NIR laser for ablation (Fig.2a ). The imaging branch of the setup consists of an inverted Nikon Eclipse Ti microscope (Nikon Instruments) equipped with a high NA water imersion objective (Plan Apo $60^{x}$, NA 1.2,Nikon) and a Yokogawa spinning disk unit (CSU-X1, Yokogawa Electric), with $50 \mu \mathrm{m}$ pinhole size. Two excitation lasers $(488 / 561 \mathrm{~nm}, 20 \mathrm{~mW}$, coherent OBIS LX) are combined with a dichroic mirror (561 nm laser BrightLine $®$ single-edge laser dichroic beamsplitter, Semrock) and directed towards the Yokogawa unit after a $10^{x}$ expansion. Fluorescence emitted by the sample and spatially filtered to retrieve sectioning by the spinning disc unit is imaged onto a sCMOS camera (pco.edge, PCO AG, Germany) mounted on the microscope left side port, resulting in a pixel size of $98 \mathrm{~nm}$. Z-scanning is achieved by moving the Z-Pizeo stage (Nano-Z stage, from Mad City Labs). Imaging is controlled in Matlab via a $\mu$ Manager $^{26} /$ Matlab interface. Synchronization with laser ablation is performed with an input/output data acquisition card (USB-6251, National Instruments).

\section{Laser ablation experiment}

Laser-ablation experiments are performed with a near infrared $\sim 130$ fs mode-locked Ti: sapphire laser (YLMO $930 \pm 10 \mathrm{~nm}$, MenloSystems) operating at $130 \mathrm{MHz}$. The laser is first expanded, then reflected by a galvanometer-based laser scanning device (6215 H, Cambridge Technology Enterprises) for steering in the sample. The mid-point of the galvanometer mirrors s then relayed onto the back focal plane of the objective with a pair of achromatic doublets which also provide a $4^{x}$ magnification with the focal length $f_{1}=75 \mathrm{~mm}$ and $f_{2}=300 \mathrm{~mm}$. The laser is then focused by the microscope objective, delivering pulse peak power density near $7.42 \times 10^{12} \mathrm{~W} \cdot \mathrm{cm}^{-} 2$. Two-dimensional laser ablation was obtained by scanning the laser beam over the focal plane with the help of the galvo-scanner. The three-dimensional ablation was achieved by moving the Z-Piezo stage (Nano-Z stage, from Mad City Labs ) and the galvano scanner along the X,Y and Z directions simultaneously (Fig. 4a,b). A data acquisition card (USB-6251, National Instruments) along with Matlab software and $\mu$ Manager is used for data acquisition 
as well as for controlling the scan mirrors, shutter and Z-stage, all devices were synchronized by the external trigger that we calculated according to the ablative region.

\section{Software and instrument synchronisation}

To enable interactive manipulations of the laser-tissue, a custom-written Graphical user interface (MATLAB) has been developed, which allows the user to perform different ablative pattern (point,linear,circular and 3D circular) directly on the target sample. To select the location of ablation, the total surface is estimated using the procedure described below. The user can then interactively manipulate a 3D rendering of the tissue, generated by projecting signal of the adherens plane onto the 3D surface, to define the pattern of ablation. Upon selection of the ablation spot/region, the program re-evaluates the surface of interest in a small window around the region of ablation (see below). It then computes the exact 3D trajectory of the focal spot so that it is as close as possible from desired trajectory (line, circle) while being confined to the curved surface (see below). Synchronized signals are then generated and sent via the DAQ board to control the galvano-mirrors, the $\mathrm{Z}$ piezoelectric stage, laser and camera triggers.

\section{Non-linear imaging and fractional Lissajous scans}

A femtosecond $(\sim 140 \mathrm{fs})$ Ti:Sapphire pulsed laser (Chameleon, Coherent) tuned to $\sim 930 \mathrm{~nm}$ is used as a the excitation for the two-photon fluorescence imaging. The laser illuminates a pair of galvanometer scanning mirrors (6215HM60, Cambridge Technology). In turn, scanning mirrors are conjugated to the back focal plane of an objective lens (CFI Plan Apochromat Lambda $20^{x}$ NA 0.75 , Nikon) that is mounted on commercial microscope stand (Eclipse Ti-U, Nikon) with piezo drive (PIFOC, Physik Instrumente) for the precise $\mathrm{Z}$ position control. The same objective lens is used to collect the fluorescence signal generated in a sample (epi geometry) and this signal is registered by a photo-multiplier tube (R9110, Hamamatsu). A set of dielectric spectral filters are used to separate the fluorescence radiation at the spectral range of $510-540 \mathrm{~nm}$ from the excitation wavelength: a dichroic mirror T770SPXR (AHFanalysentechnik, AG), a shortpass filter (ET750sp-2p8, Chroma Technology.) and a bandpass filter (510AF23 XF3080, Omega Optical incorporated). Signal acquisition and galvanometer mirrors are synchronized and controlled by an acquisition card (DAQ USB-6351, National Instruments) with use of a custom developed MATLAB code. Change of the deflection angle of the galvanometer mirrors causes the focused beam translation in the sample. The focus trajectories are based on Lissajous curves and defined the following way:

$$
\begin{aligned}
& X=\frac{X^{\max }}{2} * \sin (m t) \\
& Y=\frac{Y^{\max }}{2} * \sin \left(n t+\frac{\pi}{2}\right)
\end{aligned}
$$

where $X^{\max }$ and $Y^{\max }$ is the field of view size in the $x$ and $y$ direction, respectively, $t$ is a parameter that varies from 0 to $2 \pi$, $n$ and $m$ are integer parameters that define the sparsity of the scans. Several pairs of of $n$ and $m$ are used during acquisition depending on how dense the required scan is: $[n=41, m=43],[n=71, m=73],[n=101, m=103]$.

\section{Identification of a surface of interest from a fractional prescan.}

We adapt the surface estimation strategy of Abouakil et al. ${ }^{30}$, which we detail here for sake of completeness. The surface of interest is estimated from the sampling of only a small fraction of the voxels-space, which we call $\Omega_{0}$. When using high-content 3D-data from the spinning disc, $\Omega_{0}$ is determined by randomly picking voxels in the full volume of acquisition (typically $0.1 \%$ of the voxels). When using the lissajou scans with the NIR laser, $\Omega_{0}$ is the full Lissajou trajectory as it is already a fractional sampling of space. We first correct spatial inhomogeneities of the background signal by introducing a normalized signal,

$$
\widehat{r}_{\left[\Omega_{0}\right]}(x, y, z)=\frac{s(x, y, z)-\widehat{a}_{\left[\Omega_{0}\right]}(x, y, z)}{\widehat{a}_{\left[\Omega_{0}\right]}(x, y, z) \widehat{\sigma}_{\left[\Omega_{0}\right]}}
$$

where $s(x, y, z)$ is the measured signal, $\widehat{a}_{\left[\Omega_{0}\right]}(x, y, z)$ is an estimation of spatial inhomogeneities of the background and $\widehat{\sigma}_{\left[\Omega_{0}\right]}$ is an estimation of the standard deviation of $s(x, y, z) / \widehat{a}_{\left[\Omega_{0}\right]}(x, y, z)$ on the background. Both $\widehat{a}_{\left[\Omega_{0}\right]}(x, y, z)$ and $\widehat{\sigma}_{\left[\Omega_{0}\right]}$ are determined using only the voxels in $\Omega_{0}$. Through this normalization, the histogram of $\widehat{r}_{\left[\Omega_{0}\right]}$ falls down on an heavy-tailed Gaussian-like distribution in the case of the spinning disc data (Fig. S2). Assuming the gaussian curve corresponds to the background and the heavy tail to the bright points, we set a threshold $T$ from a chosen probability of false alarm (pfa), such that $\operatorname{pfa}=\frac{1}{2}[1-\operatorname{erf}(T / \sqrt{2})]$, where erf is the error function. We chose a pfa of $1 \%$, which corresponds to $T \approx 2.33$. The intensity distribution from the two-photon Lissajou scans is quite distinct from a Gaussian curve or heavy tailed Gaussian. Although further studies on hte noise characteristic could be performed, we found empirically that $T \approx 8$ worked for most instances. 
In a second step, we interpolate the epithelial surface modeled as $z=Z(x, y)$, using the detected bright points. To cope with noise on the location of bright points and outliers, we use local second order polynomial fits of the bright point $\mathrm{z}$-coordinates, combined with RANSAC-based outlier removal ${ }^{33}$. When surface estimation is performed in a small region of interest, around the ablated region for example, only one window is used (Fig. 6a), leading to one polynomial fit of the form $z=Z_{s}(x, y)$ where:

$$
Z_{s}(x, y)=c_{1}+c_{2} y+c_{3} y^{2}+c_{4} x+c_{5} x y+c_{6} x^{2}
$$

with $c_{1}, \ldots, c_{6}$ the 6 parameters of the surface that have been estimated.

When estimating surface over large regions of interest, for example and entire field of view $(\sim 100 \mu \mathrm{m})$, the fits are estimated in overlapping windows (Fig. 6b) and are then fused. To fuse the estimations in the overlapping regions, we do not use the parameters of the quadratic fits (eq. 4), but instead filter the corresponding inliers from the RANSAC approach. More specifically, bright points that are inliers in all of the overlapping quadratic fits are preserved, while those that are inliers in only a fraction $(1 / 4,2 / 4$ or $3 / 4)$ of the overlapping windows are discarded. The surface is then estimated through the interpolation of the remaining inliers using a simple bi-cubic harmonic spline interpolation ${ }^{43}$. Figure S1 shows the successive steps of surface estimation for the tissue of Fig. 6. Once the surface of interest $\widehat{Z}(x, y)$ is determined, it can be converted into a thin shell by setting a thickness along $z$ of $\varepsilon=3 \mu \mathrm{m}$, which is slightly larger than the thickness of adherens junctions.

\section{Estimation of the ablation trajectory in a tangent plane}

When performing circular ablations, it is necessary for the trajectory to be circular locally on the surface of the tissue. One therefore cannot just impose a circular trajectory with the galvanometers (xy-plane) and project it onto the surface of the tissue. We therefore compute a trajectory which is included in the surface and as much as possible parallel to the local tangent plane and almost circular. The starting point is the local estimation of the surface with a quadratic form, the outcome of the surface estimation step with one window. A point with coordinates $(x, y, z)$ located on this surface is defined so that $z=Z_{s}(x, y)$, given by eq. 4. For a given point $P_{0}$ on this surface, with coordinates $\left(x_{0}, y_{0}, z_{0}=Z_{s}\left(x_{0}, y_{0}\right)\right.$, let us define $\mathscr{P}_{0}$ the local plane that is tangent to this surface at point $P_{0}$. Two tangent vectors $\vec{V}_{x}$ and $\vec{V}_{y}$ lying on $\mathscr{P}_{0}$ and a vector $\vec{V}_{n}$ normal to $\mathscr{P}_{0}$ are then given by

$$
\vec{V}_{x}=\left[\begin{array}{c}
1 \\
0 \\
c_{4}+c_{5} y_{0}+2 c_{6} x_{0}
\end{array}\right], \vec{V}_{y}=\left[\begin{array}{c}
0 \\
1 \\
c_{2}+2 c_{3} y_{0}+c_{5} x_{0}
\end{array}\right], \vec{V}_{n}=\left[\begin{array}{c}
-c_{4}-c_{5} y_{0}-2 c_{6} x_{0} \\
-c_{2}-2 c_{3} y_{0}-c_{5} x_{0} \\
1
\end{array}\right]
$$

The circular trajectory of radius $R$ lying on the plane $\mathscr{P}_{0}$ can be defined by the set of points $M_{0}(\theta)$ such that

$$
\overrightarrow{O M}(\theta)=\overrightarrow{O P} P_{0}+R \cos \theta \frac{\vec{V}_{x}}{\left\|V_{x}\right\|}+R \sin \theta \frac{\vec{V}_{y}}{\left\|V_{y}\right\|}
$$

while varying $\theta$ from 0 to $2 \pi$ and with $O$ the axis origin. Nevertheless, this trajectory is not contained on the quadratic surface (unless $c_{3}=c_{5}=c_{6}=0$ ). We thus project these points $M_{0}(\theta)$ on the quadratic surface, orthogonally to the plane $\mathscr{P}_{0}$ (i.e. in direction $\left.\vec{V}_{n}\right)$. With defining $M_{h}(\theta)$ the translation of point $M_{0}(\theta)$ of length $h$ and in direction $\vec{V}_{n}$, i.e.

$$
\overrightarrow{O M}_{h}(\theta)=\overrightarrow{O M_{0}}(\theta)+h \frac{\vec{V}_{n}}{\left\|V_{n}\right\|}=\overrightarrow{O P}+h \frac{\vec{V}_{n}}{\left\|V_{n}\right\|}+R \cos \theta \frac{\vec{V}_{x}}{\left\|V_{x}\right\|}+R \sin \theta \frac{\vec{V}_{y}}{\left\|V_{y}\right\|}
$$

the trajectory projected on the surface can then be obtained as the set of points $M_{h_{\theta}}(\theta)$ where for each angle $\theta, h_{\theta}$ is defined as the value of $h$ for which $M_{h}(\theta)$ lies on the quadratic surface, i.e. so that

$$
\overrightarrow{O M_{h_{\theta}}}(\theta) \cdot \vec{k}=Z_{s}\left(\overrightarrow{O M}_{h_{\theta}}(\theta) \cdot \vec{i}, \overrightarrow{O M}_{h_{\theta}}(\theta) \cdot \vec{j}\right)
$$

where $\vec{i}, \vec{j}, \vec{k}$ are unit basis vectors along $x, y, z$ directions. This trajectory now lies on the quadratic surface and corresponds to a circle of center $P_{0}$ and radius $R$ when projected orthogonally on the plane $\mathscr{P}_{0}$ tangent to the surface at $P_{0}$. Such a projection introduces minimal distortions from a perfect circle, while ensuring that the laser focus remains as close as possible from the adherens plane, for an optimal ablation (see Fig. S3).

\section{Preparation of biological samples}

All observations were performed on living Drosophila tissues, using an E-cadherin:GFP knock-in to image adherens junctions ${ }^{44}$. We imaged wing imaginal discs as in LeGoff et al. ${ }^{34}$; Living tissues were dissected from late third instar larva using a stereo-microscope, and cultured in a drop of Grace's insect medium (sigma) in a glass bottom petri-dish. All experiments are performed at $22^{\circ} \mathrm{C}$. 


\section{Acknowledgements}

We thank Sophie Brasselet, Dana Bruner and Morgane Chauvet for help and discussions on the project.

This work was funded by the following agencies: Agence Nationale de la Recherche (ANR-18-CE13-028, ANR-17-CE30-0007) ; Excellence Initiative of Aix-Marseille University - A*Midex (capostromex), a French Investissements d'Avenir programme; The project leading to this publication has received funding from the «Programme d'Investissements d'Avenir » of the French Government, managed by the French National Research Agency (ANR-16-CONV-0001, ANR21-ESRE-0002), and from Excellence Initiative of Aix-Marseille University - A*MIDEX.

HM thanks the support of the China Scholarship Council(CSC).

\section{Author contributions statement}

LLG conceived the experiments. HM built the apparatus with the help of MS, HM conducted most experiments except for two photon microscropy which was conducted by HM and DN. HM, FG, and LLG designed and performed data analysis. HM and LLG wrote the paper. All authors reviewed the manuscript.

\section{Competing interests}

The authors declare no competing interest.

\section{References}

1. Pantazis, P. \& Supatto, W. Advances in whole-embryo imaging: a quantitative transition is underway. Nat. Rev. Mol. Cell Biol. 15, 327-339 (2014).

2. Belin, B. J., Goins, L. M. \& Mullins, R. D. Comparative analysis of tools for live cell imaging of actin network architecture. Bioarchitecture 4, 189-202 (2014).

3. Latorre, E. et al. Active superelasticity in three-dimensional epithelia of controlled shape. Nature 563, 10.1038/ s41586-018-0671-4 (2018).

4. Hur, S. et al. Traction force microscopy for understanding cellular mechanotransduction. BMB Reports 53, 74-081, 10.5483/BMBRep.2020.53.2.308 (2020).

5. Kong, W. et al. Experimental validation of force inference in epithelia from cell to tissue scale. Sci. Reports 9, 10.1038/ s41598-019-50690-3 (2019).

6. Vasan, R., Maleckar, M. M., Williams, C. D. \& Rangamani, P. Dlite uses cell-cell interface movement to better infer cell-cell tensions. Biophys. J. 117, 1714-1727, https://doi.org/10.1016/j.bpj.2019.09.034 (2019).

7. Roffay, C., Chan, C. J., Guirao, B., Hiiragi, T. \& Graner, F. Inferring cell junction tension and pressure from cell geometry. Development 148, dev192773 (2021).

8. Stabley, D., Jurchenko, C., Marshall, S. \& Salaita, K. Visualizing mechanical tension across membrane receptors with a fluorescent sensor. Nat. methods 9, 64-7, 10.1038/nmeth.1747 (2012).

9. Houser, J., Hayden, C., Thirumalai, D. \& Stachowiak, J. A förster resonance energy transfer-based sensor of steric pressure on membrane surfaces. J. Am. Chem. Soc. 142, 20796-20805, 10.1021/jacs.0c09802 (2020).

10. Campàs, O. et al. Quantifying cell-generated mechanical forces within living embryonic tissues. Nat. methods 11, 183-189 (2014).

11. Dolega, M. et al. Cell-like pressure sensors reveal increase of mechanical stress towards the core of multicellular spheroids under compression. Nat. communications 8, 1-9 (2017).

12. Hutson, M. S. et al. Forces for morphogenesis investigated with laser microsurgery and quantitative modeling. Science 300, 145-149 (2003).

13. Smutny, M., Behrndt, M., Campinho, P., Ruprecht, V. \& Heisenberg, C.-P. Uv laser ablation to measure cell and tissuegenerated forces in the zebrafish embryo in vivo and ex vivo. Methods molecular biology (Clifton, N.J.) 1189, 219-35, 10.1007/978-1-4939-1164-6_15 (2015).

14. Rauzi, M., Verant, P., Lecuit, T. \& Lenne, P.-F. Nature and anisotropy of cortical forces orienting drosophila tissue morphogenesis. Nat. cell biology 10, 1401-1410 (2008).

15. Izquierdo, E., Quinkler, T. \& De Renzis, S. Guided morphogenesis through optogenetic activation of rho signalling during early drosophila embryogenesis. Nat. communications 9, 1-13 (2018). 
16. Guglielmi, G., Barry, J. D., Huber, W. \& De Renzis, S. An optogenetic method to modulate cell contractility during tissue morphogenesis. Dev. cell 35, 646-660 (2015).

17. Colombelli, J., Grill, S. W. \& Stelzer, E. H. Ultraviolet diffraction limited nanosurgery of live biological tissues. Rev. Sci. Instruments 75, 472-478 (2004).

18. Supatto, W. et al. In vivo modulation of morphogenetic movements in drosophila embryos with femtosecond laser pulses. Proc. Natl. Acad. Sci. 102, 1047-1052 (2005).

19. Rauzi, M. Probing tissue interaction with laser-based cauterization in the early developing drosophila embryo. Methods cell biology 139, 153-165, 10.1016/bs.mcb.2016.11.003 (2017).

20. Campàs, O. A toolbox to explore the mechanics of living embryonic tissues. Semin. cell amp; developmental biology 55, 119-130, 10.1016/j.semcdb.2016.03.011 (2016).

21. Schajer, G. S. Advances in hole-drilling residual stress measurements. Exp. mechanics 50, 159-168 (2010).

22. Langer, K. Zur anatomie und physiologie der haut. über die spaltbarkeit der cutis. Sitzungsbericht der Math. Cl. der Wiener Kaiserlichen Acad. der Wissenschaften 44 (1861).

23. Bonnet, I. et al. Mechanical state, material properties and continuous description of an epithelial tissue. J. The Royal Soc. Interface 9, 2614-2623 (2012).

24. Ben Amar, M., Qiuyang-Qu, P., Vuong-Brender, T. T. K. \& Labouesse, M. Assessing the contribution of active and passive stresses in c. elegans elongation. Phys. review letters 121, 268102 (2018).

25. Guillot, C. \& Lecuit, T. Mechanics of epithelial tissue homeostasis and morphogenesis. Science 340, 1185-1189 (2013).

26. Edelstein, A. D. et al. Advanced methods of microscope control using $\mu$ manager software. J. biological methods 1 (2014).

27. de Medeiros, G. et al. Cell and tissue manipulation with ultrashort infrared laser pulses in light-sheet microscopy. Sci. reports 10, 1-12 (2020).

28. Sugimura, K., Lenne, P.-F. \& Graner, F. Measuring forces and stresses in situ in living tissues. Development 143, 186-196 (2016).

29. Ackermann, J., Qu, P.-Q., LeGoff, L. \& Ben Amar, M. Modeling the mechanics of growing epithelia with a bilayer plate theory. The Eur. Phys. J. Plus 137, 1-29 (2022).

30. Abouakil, F. et al. An adaptive microscope for the imaging of biological surfaces. Light. Sci. Appl. 10, in press (2021).

31. Beira, J. V. \& Paro, R. The legacy of drosophila imaginal discs. Chromosoma 125, 573-592 (2016).

32. Linz, N. et al. Wavelength dependence of nanosecond infrared laser-induced breakdown in water: Evidence for multiphoton initiation via an intermediate state. Phys. Rev. B 91, 10.1103/PhysRevB.91.134114 (2015).

33. Fischler, M. A. \& Bolles, R. C. Random sample consensus: a paradigm for model fitting with applications to image analysis and automated cartography. Commun. ACM 24, 381-395 (1981).

34. LeGoff, L., Rouault, H. \& Lecuit, T. A global pattern of mechanical stress polarizes cell divisions and cell shape in the growing drosophila wing disc. Development 140, 4051-4059 (2013).

35. Mao, Y. et al. Differential proliferation rates generate patterns of mechanical tension that orient tissue growth. The EMBO journal 32, 2790-2803 (2013).

36. Dye, N. A. et al. Self-organized patterning of cell morphology via mechanosensitive feedback. Elife 10, e57964 (2021).

37. Rauzi, M. \& Lenne, P.-F. Cortical forces in cell shape changes and tissue morphogenesis. Curr. topics developmental biology 95, 93-144, 10.1016/B978-0-12-385065-2.00004-9 (2011).

38. Grewe, B. F., Voigt, F. F., van't Hoff, M. \& Helmchen, F. Fast two-layer two-photon imaging of neuronal cell populations using an electrically tunable lens. Biomed. optics express 2, 2035-2046 (2011).

39. Shain, W. J., Vickers, N. A., Goldberg, B. B., Bifano, T. \& Mertz, J. Extended depth-of-field microscopy with a high-speed deformable mirror. Opt. letters 42, 995-998 (2017).

40. Tuma, T., Lygeros, J., Kartik, V., Sebastian, A. \& Pantazi, A. High-speed multiresolution scanning probe microscopy based on lissajous scan trajectories. Nanotechnology 23, 185501 (2012).

41. Deguchi, T. et al. Volumetric lissajous confocal microscopy with tunable spatiotemporal resolution. Biomed. Opt. Express 11, 6293-6310 (2020). 
42. Olivier, N. et al. Cell lineage reconstruction of early zebrafish embryos using label-free nonlinear microscopy. Science 329, 967-971 (2010).

43. Sandwell, D. T. Biharmonic spline interpolation of geos-3 and seasat altimeter data. Geophys. Res. Lett. 14, 139-142, https: //doi.org/10.1029/GL014i002p00139 (1987). https://agupubs.onlinelibrary.wiley.com/doi/pdf/10.1029/GL014i002p00139.

44. Huang, J., Zhou, W., Dong, W., Watson, A. M. \& Hong, Y. Directed, efficient, and versatile modifications of the drosophila genome by genomic engineering. Proc. Natl. Acad. Sci. 106, 8284-8289 (2009). 


\section{Supplementary material}
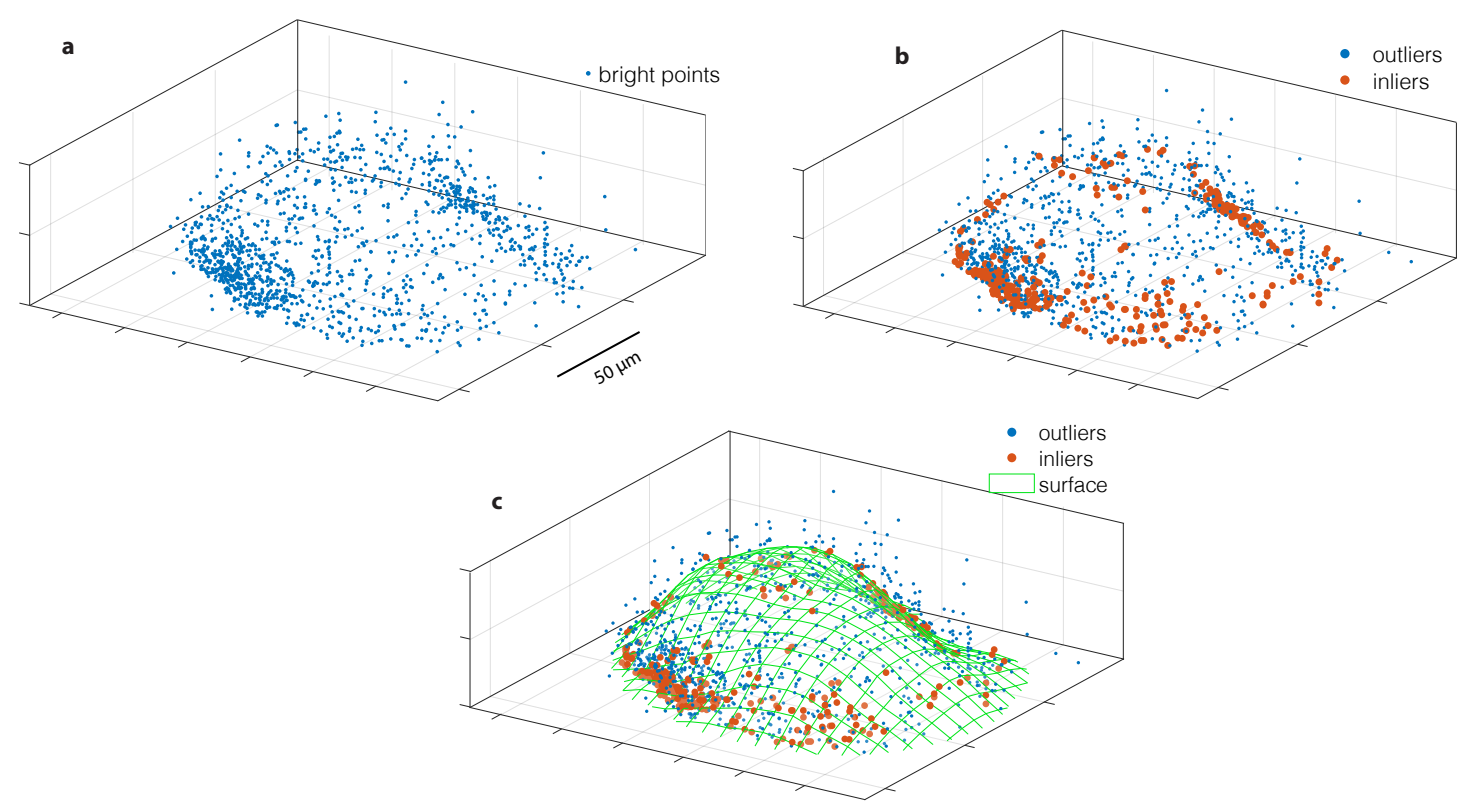

Figure S1. Steps of surface estimation. All data are taken from the surface estimation with lissajou scans from Figure 6c-f.(a) Bright points detection with a simple threshold on the Lissajou prescan after correction for inhomogeneities (eq. 3). (b) classification of bright points into inliers and outliers using ransac polynomial fits. (c) surface estimation form the interpolation of inliers using spline fitting. 


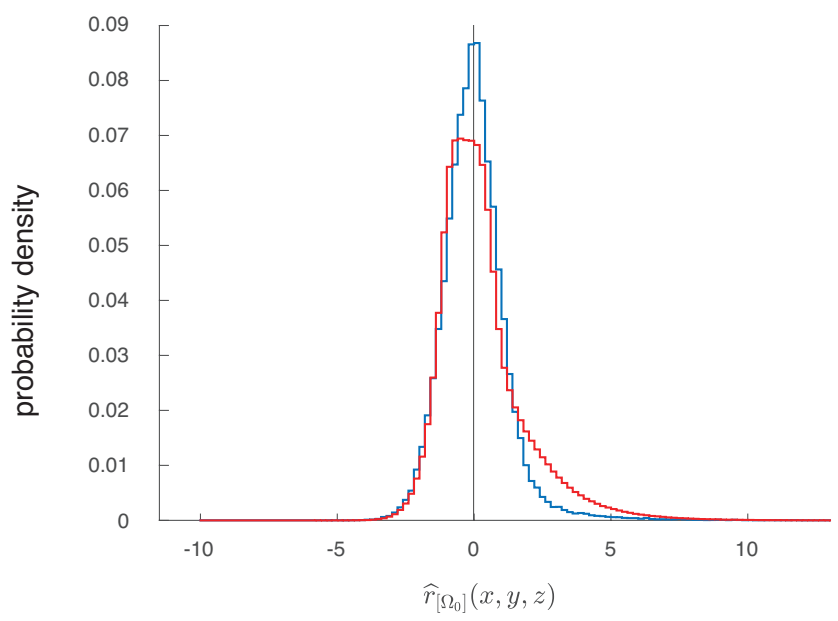

Figure S2. Intensity distributions after correction of inhomogeneities. Distribution of the normalized signal introduced by equation * in linear fluorescence stacks and in the two photon fluorescence case. In the spinning disc case (in blue), the distribution is symmetric and gaussian distributed with a small heavy tail at high intensities. In the two photon case (red), the distribution is much less symmetric. 


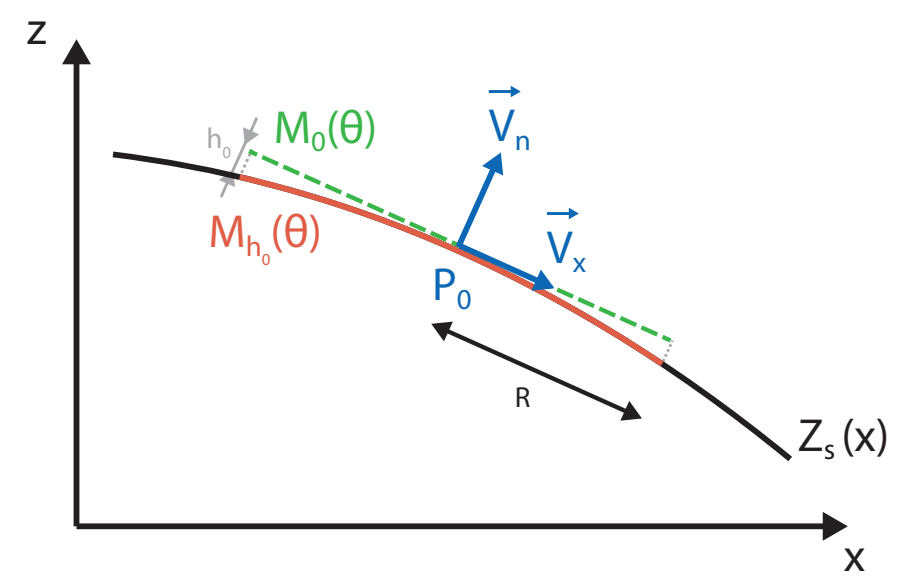

Figure S3. Geometry of ablation on a curved surface. (a) Quadratic surface with local tangent and normal in blue. The green dotted line outlines a strictly circular trajectory, parallel to the local tangent. We project this ring on the surface $Z_{s}$ (red curve) for an optimal ablation process. The projection is performed along the normal of the surface $\left(V_{n}\right)$. One point of the circular trajectory $\left(M_{\theta}(\theta)\right)$ and its projection $\left(M_{h_{\theta}}(\theta)\right)$, discussed in the method, are also shown. 